\title{
Exogenous steroid hormones stimulate full development of autonomous endosperm in Arabidopsis thaliana
}

\author{
Joanna Rojek ${ }^{1 *}$, Łukasz Pawełko', Małgorzata Kapusta', Aleksandra Naczk², Jerzy Bohdanowicz' \\ 1 Department of Plant Cytology and Embryology, University of Gdańsk, Wita Stwosza 59, 80-308 Gdańsk, Poland \\ ${ }_{2}^{2}$ Department of Molecular Evolution, University of Gdańsk, Wita Stwosza 59, 80-308 Gdańsk, Poland
}

\begin{abstract}
Most flowering plants, including important crops, require double fertilization to form an embryo and endosperm, which nourishes it. Independence from fertilization is a feature of apomictic plants that produce seeds, from which the plants that are clones of the mother plant arise. The phenomenon of apomixis occurs in some sexual plants under specific circumstances. Since the launch of a fertilization-independent mechanism is considered a useful tool for plant breeding, there have been efforts to artificially induce apomixis. We have been able to produce fertilization-independent endosperm in vitro in Arabidopsis over the last few years. This paper demonstrates the methods of improving the quality of the endosperm obtained using plant and mammalian steroid hormones. Additionally, it shows the study on the autonomous endosperm (AE) formation mechanism in vitro.

This paper examines the effect of exogenous steroid hormones on unfertilized egg and central cell divisions in culture of unpollinated pistils of Arabidopsis Col-0 wild-type and fie-1 mutant. All media with hormones used (estrone, androsterone, progesterone, and epibrassinolide) stimulated central cell divisions and fertilization-independent endosperm development. The stages of AE development followed the pattern of Arabidopsis thaliana wild type after fertilization. Subsequent stages of AE were observed from 2-nuclear up to cellular with the most advanced occurring on medium with 24-epibrassinolide and progesterone. The significant influence of mammalian sex hormones on speed of AE development and differentiation was noticed. Using restriction analysis, the changes in methylation of FIE gene was established under in vitro condition. The authors of this paper showed that Arabidopsis thaliana has a high potency to fertilization-independent development.
\end{abstract}

Keywords: Arabidopsis; apomixis; autonomous endosperm; steroid hormones; DNA methylation

\section{Introduction}

\section{Autonomous endosperm}

In sexually reproducing Angiosperms such as Arabidopsis, double fertilization is required for the formation of the embryo and endosperm from fertilized egg cell and central cell, respectively [1]. The other reproductive strategy, devoid of meiosis (or meiosis is disturbed, depending on apomixis type) and fertilization of the egg cell, is apomixis, where the progeny are clones of the mother [2]. Molecular mechanism of apomixis has been investigated for many years, and several genes have been discovered that may contribute to the initiation of apomictic development. According to the recent theory, the mechanism of apomixis was developed from sexual reproduction by hybridization and polyploidization, under the influence of the environment [3]. Research on apomixis is focused on

\footnotetext{
* Corresponding author. Email: joanna.rojek@biol.ug.edu.pl
}

Handling Editor: Elżbieta Bednarska-Kozakiewicz the mechanisms responsible for the disruption or inhibition of meiosis in megaspore mother cell, and on examination of the factors stimulating unfertilized egg cell and central cell development. dyad, mel1 (argonaute) mutations allow the formation of unreduced gametes, thus imitating diplospory $[4,5]$. Among the genes controlling seed development, FIS genes are well known and their products form part of the polycomb repressive complex 2 (PRC2). The FIS-PRC2 cooperates closely with MET1 and controls the expression of genes related to seed development [6]. In fis mutants the autonomous endosperm (AE) is partly developed despite being fertilization-independent, resembling the apomictic pathway. In addition, a combination of fis and met 1 mutations allows, in the absence of fertilization, further (more advanced) development of autonomous endosperm in planta and more frequent occurrence of autonomous endosperm (fie-1 mutant plants carrying an MET1 antisense silencing construct [7]; mea-1/MEA; met1-3/MET1 mutant [6]). A similar phenomenon was observed in in vitro cultured unfertilized ovules (inside ovaries) of wild Arabidopsis genotypes [8,9] and in met1 mutant, in which AE induction 
does not or was very sporadically noted in planta [10]. AE development was studied on modified MS medium (with $6 \%$ sucrose) supplied with auxin and cytokinin [10].

\section{Steroid hormones}

Brassinosteroids (BRs) have been discovered in more than 58 plant species in stems, roots, buds, pollen grains, and seeds [11]. Their content in plant cells is very low, which corresponds with the physiological activity of the hormone in concentrations of about 1000 times smaller than other previously known hormones [12]. BRs regulate root development, anther and pollen development and formation, stem elongation, vascular tissue differentiation and cellulose biosynthesis. Furthermore, the physiological, cellular and molecular mechanisms by which BRs regulate plant development suggest that the biological functions of BRs are far beyond promoting cell elongation [13]. BRs are involved in plant reproductive growth, specifically in regulating male fertility $[14,15]$. In addition, fruit size was increased with the exogenously applied BR or by overexpression of BR synthetic genes in cucumber and tomato, indicating that fruit development $[16,17]$ and flowering [18] are regulated by BRs. They also contribute in response to various stresses, such as very low or very high temperatures, drought, high osmotic pressure, and pathogen attack [19,20].

In the field of in vitro cultures, where many species and varieties require specific composition of the media, there is a high demand for new growth and development regulators. BRs are being increasingly used as a replacement or together with the previously most used auxins and cytokinins and their derivatives. BR support growth of callus and stimulate the embryogenesis and organogenesis in many species, including cultivars [20]. In suspension cultures of Arabidopsis thaliana 24-epibrassinolide replaced the cytokinin action in the culture media used for multiplication cell proliferation [21].

Male and female mammalian sex hormones (MSHs), which include androgens, estrogens and progesterone are involved in reproduction in mammals. The presence of sex hormones in plants was postulated as early as the 20th century [14], and although the presence of a majority of them is questionable, literature confirms the presence of mammalian steroids in 128 species from over 50 families [22]. In 2005, The membrane protein MSBP1 was discovered in the cells of Arabidopsis, which resembles the animal receptor binding progesterone and binds the plant progesterone and other plant steroid hormones [23]. MSBP1 functions as negative regulator of cell elongation in Arabidopsis [23].

Selected mammalian steroids, exogenously deposited, stimulate plant cell division, the maturation of pollen as well as growth and flowering [24]. Progesterone, estrone and androsterone induce in vitro flowering in Arabidopsis thaliana [25]. Exogenous application of MSHs activate the oxidative enzymes under non-stress conditions [26]. The latest results obtained in spring wheat after drought treatment [24] suggest that progesterone and its putative receptors contribute in processes regulating plant interactions with the environment. Thus, exogenous application of MSHs may be used to increase the plant resistance to negative environmental conditions.

\section{5-azacytidine as demethylating factor}

5 -azacytidine $(5$-azaC) is an analogue of pyrimidine nucleoside, which inhibits CG and methylation, and induces hypomethylation in plant and animal genome [27,28]. The treatment of plant and animal cells with 5-azaC solutions leads to direct (by incorporating analogue of cytosine into DNA during replication) and indirectly (by inhibiting DNA methyltransferase) DNA demethylation.

5 -azaC is one of the most widely used demethylating agents in experimental systems. In animal cultures of established cell lines, 5-azaC is used, inter alia, to investigate the effects of the chemotherapeutic agent in the treatment of tumors [29-31]. In plants, 5-azaC is helpful in understanding the mechanisms that control the gene silencing [32]. Active DNA demethylation occurs in plant genome in response to biotic and abiotic stresses suggesting that DNA methylation/demethylation control the mechanisms of plant stress response [33]. A study by Ruiz-Garcia and coauthors [34], using AFLP technique has shown that 5-azaC causes drastic demethylation. The effect of demethylation was the greater, the higher concentration of 5-azaC was applied to Arabidopsis seedlings. Treatment of seeds and seedlings of certain Arabidopsis mutants and Thlaspi arvense with 5-azaC induces premature flowering [35]. In turn, overexpression of an antisense transcript of DNA methyltransferase [antisense-cDNA methyltransferase (MET1)] induces flowering and reduces FLC gene expression, which is one of the main inhibitors of flowering in Arabidopsis [32]. The use of 5-azaC as DNA demethylating agent in experimental conditions allows the study on the regulation of the activity of the genes responsible for flowering (FLC, VIN, VRN) and seed development (like FIE [36]) through the mechanism of methylation/demethylation [32].

Some questions were raised since successfully induced process of the fertilization-independent central cell development into endosperm by the authors of this paper in Arabidopsis. (i) If the endosperm in wild genotypes of Arabidopsis thaliana appears in all (up to now) of media used is this a feature specific to this species? (ii) Is the mechanism of induction of autonomous endosperm the same, despite different ways of induction (by mutation or by the action of the in vitro condition)? Is this mechanism also the same in apomicts? (iii) Is it possible to initiate in vitro or in planta development of haploid embryo in wild genotypes of Arabidopsis?

We started a wide range of planned experiments based on the knowledge of the crucial role of FIS-POLYCOMB group and MET1 in sexual reproduction, and the results of the first part of the experiments are showed in this paper.

Using new steroid hormones and demethylating factor in the experiment to investigate Arabidopsis thaliana unfertilized central cell and the egg cell divisions in unpollinated in vitro cultured ovules, following elements were expected: the induction of autonomous endosperm formation, increase in the frequency of ovules with $\mathrm{AE}$ and advancement in $\mathrm{AE}$ development. This research confirms the supposed genetic ability of sexually reproducing Arabidopsis to endosperm development according to autonomous apomictic pattern. 


\section{Material and methods}

\section{Plant material and growth conditions}

Plants were grown from Col-0 seeds [NASC ID (ABRC): 22625] and fie seeds (fie-1; obtained from Prof. Nir Ohad; unavailable from NASC or ABRC [37]), in a glasshouse under cold fluorescent light $\left(120 \mu \mathrm{mol} / \mathrm{m}^{2} / \mathrm{s}\right)$ at $21^{\circ} \mathrm{C}$ and $70 \%$ humidity under long days (16 h light) until flowering and seed setting.

The fie genotypes were verified by molecular analysis; additionally, fie genotypes were recognized by silique phenotype (see, e.g. [38]).

The PCR protocol for distinguishing the WT FIE allele from the mutant allele 547 was based on amplification of genomic DNA with PCR, followed by restriction analysis. The mutant allele (line 547 TTTAAA) was restricted with DraI, while the WT allele (TCTAAA) was not restricted (one band of $866 \mathrm{bp}$ from the WT allele and another two bands of $374 \mathrm{bp}$ and $492 \mathrm{bp}$ from the mutant allele after restriction with DraI). Primers were as follows: 3143 (R) 5' CCTATATGGCAACAGAAAAT 3' and 2277 (F) 5' GCTTGTGGTTCGTTTGTATG 3' (Integrated DNA Technologies - ITD, Inc.; Genomed, Poland).
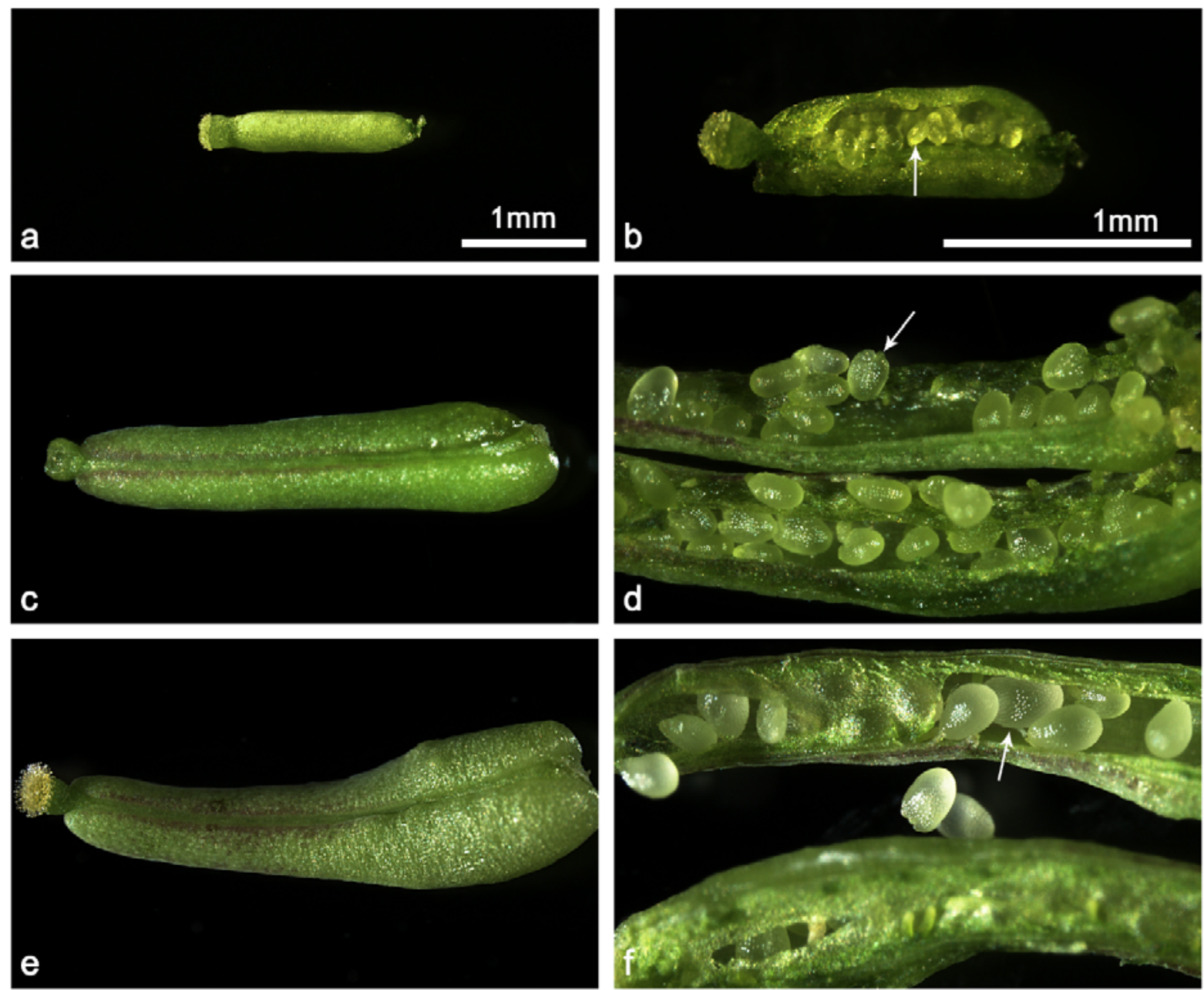

Fig. 1 In vitro culture of unpollinated pistils on selected steroid hormones induce grow and development. a-b The unpollianted pistil of Arabidopsis thaliana (Col-0) at inoculation. c-f At 7th day; significant enlargement of pistils and ovules (arrows) inside ovaries on M2 medium with androsterone (c-d) and M3 medium with epiBL (e-f). Note most enlarged ovaries and ovules on M3 (f). Scale bar at $\mathbf{a}$ fits also for $\mathbf{c}$ and $\mathbf{e}$. Scale bar at $\mathbf{b}$ fits also for $\mathbf{d}$ and $\mathbf{f}$. 
Tab. 1 The induction of autonomous endosperm (AE) in unpollinated ovules of Arabidopsis thaliana on media with steroid hormones and on hormone-free medium (M1 - control).

\begin{tabular}{|c|c|c|c|c|c|c|c|c|}
\hline \multirow{2}{*}{\multicolumn{2}{|c|}{ Medium }} & \multicolumn{3}{|c|}{$\begin{array}{l}\text { No. of analyzed viable explants at } 7 \text { th } \\
\text { day of culture }\end{array}$} & \multicolumn{4}{|c|}{ Percentage of AE induction } \\
\hline & & \multirow{2}{*}{ Ovaries } & \multirow{2}{*}{ Ovules } & \multirow{2}{*}{ Ovules/ovary ${ }^{1}$} & \multicolumn{2}{|c|}{ Ovaries (\%) } & \multicolumn{2}{|c|}{ Ovules (\%) } \\
\hline \multicolumn{6}{|c|}{ Col-0 } & & & \\
\hline M1 & MS+suc6\% & 50 & 866 & $17 \pm 0.19$ & 8 & $(16.0)$ & 8 & $(0.9)$ \\
\hline M2 & $\mathrm{MS}+$ suc6\%+Estr $1 \mu \mathrm{M}$ & 50 & 1397 & $28 \pm 0.17$ & 14 & $(28.0)$ & 19 & (1.4) \\
\hline M3 & MS+suc6\%+Andr $1 \mu \mathrm{M}$ & 50 & 1751 & $35 \pm 0.23$ & 14 & $(28.0)$ & 19 & (1.1) \\
\hline M4 & MS+suc6\%+Prog $1 \mu \mathrm{M}$ & 50 & 1700 & $34 \pm 0.19$ & 20 & $(40.0)$ & 27 & (1.6) \\
\hline M5 & MS+suc6\%+epiBL $1 \mu \mathrm{M}$ & 50 & 1400 & $28 \pm 0.37$ & 17 & $(34.0)$ & 28 & (2.0) \\
\hline \multicolumn{9}{|c|}{ fie-1/FIE } \\
\hline M1 & MS+suc6\% & 50 & 908 & $18 \pm 0.15$ & 40 & $(80.0)$ & 249 & $(27.4)$ \\
\hline M2 & MS+suc6\%+Estr $1 \mu \mathrm{M}$ & 50 & 1386 & $28 \pm 0.15$ & 28 & $(56.0)$ & 387 & $(27.9)$ \\
\hline M3 & MS+suc6\%+Andr $1 \mu \mathrm{M}$ & 50 & 1593 & $32 \pm 0.15$ & 48 & $(96.0)$ & 502 & $(31.5)$ \\
\hline M4 & MS+suc6\%+Prog $1 \mu \mathrm{M}$ & 50 & 1700 & $34 \pm 0.17$ & 42 & $(84.0)$ & 709 & (41.7) \\
\hline M5 & MS+suc6\%+epiBL $1 \mu \mathrm{M}$ & 50 & 1396 & $28 \pm 0.39$ & 50 & $(100.0)$ & 655 & $(46.9)$ \\
\hline
\end{tabular}

${ }^{1}$ Average frequency ovules per ovary $\pm S E_{m}$.

medium (MS + suc6\% + $0.1 \mathrm{mg} \mathrm{l}^{-1} \mathrm{NAA}+2 \mathrm{mg} \mathrm{l}^{-1} \mathrm{BAP}$ ). Both pistils and seedlings were cultured on M7 medium with 5 -azacytidine - demethylating factor (MS + suc $6 \%+50 \mu \mathrm{M}$ 5 -azaC). Explants cultured on 5-azaC were twice transferred on fresh medium, during the 7-days culture.

NAA, BAP and 2,4D were added to media prior to autoclaving. 5-azacytidine (Sigma) was dissolved into $1 \%$ acetic acid, stored in stock $(15 \mathrm{mg} / \mathrm{ml})$ at $-70^{\circ} \mathrm{C}$ and sterilized through $0.22 \mu \mathrm{m}$ filters (Millipore).

All cultures were maintained under a $16 \mathrm{~h}$ photoperiod at $21 \pm 3^{\circ} \mathrm{C}$. Light was supplied by cool white fluorescent tubes (avg. $70-100 \mathrm{M}$ photons $\mathrm{m}^{-1} \mathrm{~s}^{-2}$ ). Pistils were cultured for 7 days.

The viability of pistils and ovules was examined at 7 th day of culture. The morphological analyses were performed under the stereoscopic microscope. Firmness of tissues (of the ovaries and ovules), the intensity of coloration and size were compared with control material.

\section{Embryological study}

Embryological processes were analyzed in flower buds at inoculation and in unpollinated pistils cultured in vitro. Pistils cultured on different media (Tab. 1) were fixed on the 3rd, 5th, and 7th day of culture in either acetic alcohol (glacial acetic acid : $96 \%$ ethanol, 1:3, v/v), or FAA (40\% formalin : glacial acetic acid : 70\% ethanol, 5:5:90, v/v/v). Fixed material was prepared and analyzed as described by Rojek et al. [10].

\section{Statistical analysis}

The obtained percentage frequency of $\mathrm{AE}$ data were arcsine-transformed for further statistical analysis. Statistical analysis were carried out to describe: the differences in relative proportion of induction of $\mathrm{AE}$ for two study genotypes on different type of medium; the differences in relative proportion of induction of $\mathrm{AE}$ in four developmental stages on different types of medium within Col- 0 and fie-1/ FIE genotypes, respectively. Two-way ANOVA with three replications was performed separately for the following factors: (i) genotype expression (Col-0 versus fie-1/FIE) and type of medium (M1: MS + suc6\%; M2: MS + suc $6 \%+$ Estr $1 \mu \mathrm{M} ; \mathrm{M} 3: \mathrm{MS}+\operatorname{suc} 6 \%+$ Andr $1 \mu \mathrm{M} ; \mathrm{M} 4: \mathrm{MS}+$ suc6\% + Prog $1 \mu \mathrm{M}$; M5: MS + suc6\% + epiBL $1 \mu \mathrm{M}$ ); (ii) relative proportion of $\mathrm{AE}$ in four developmental stages (AE1 - fewnuclear AE; AE2 - multinuclear AE; AE3 - multinuclear $\mathrm{AE}$ just before cellularization; AE4 - cellular AE) and type of medium (M1-M5) for Col-0; (iii) relative proportion of $\mathrm{AE}$ in four developmental stages (AE1-AE4) and type of medium (M1-M5) for fie-1/FIE. To identify significant differences between treatment groups we used a post-hoc Tukey's HSD test at $P<0.05$ level (results were presented in two ways - as homogeneous groups, as well as significant differences). The increase of $\mathrm{AE}$ induction in study genotypes in different types of medium and also in four developmental stages of AE in different types of medium for Col- 0 and fie-1/FIE genotypes were illustrated by interactions graphs (Appendix S2, Appendix S3). All described analysis were performed with the program package STATISTICA v. 10 [40], according with standard procedures.

\section{DNA methylation analysis}

The degree of methylation was analyzed on FIE gene (AT3G20740.1; The Arabidopsis Information Resource TAIR database) of Col-0 in 6 experimental variants, including control (unppollinated pistils at inoculation): (i) ovaries analyzed at 7th day of culture on M1 (MS + suc6\%); (ii) on M6 (MS + suc6\% + $0.1 \mathrm{mg} \mathrm{l}^{-1} \mathrm{NAA}+2 \mathrm{mg} \mathrm{l}^{-1} \mathrm{BAP}$ ); (iii) on medium with demethylating factor (M7; MS + suc $6 \%+$ 5-azaC $50 \mu \mathrm{M}$ ); (iv) 7th-day seedlings cultured on M1, and (v) on M7 medium. 
A genomic DNA isolation was performed using standard kit (A\&A Biotechnology). Analysis was done by (i) digesting the total DNA of a pair of restriction enzymes (izoschizomers) HpaII (New England Biolabs) and MspI (Fermentas); (ii) PCR reactions using primers specific to certain locations in the studied gene. For PCR a RedTaq ${ }^{\mathrm{mm}}$ polymerase (Sigma) and $\mathrm{MgCl}$ buffer $(1.5 \mathrm{mM})$ were used. A first (FIE I - positive control; no sites of digestion) and third pair of primers (FIE III) FIE gene were selected for proper analysis of the methylation; (iii) separation of the PCR products by agarose electrophoresis. Primers for FIE (no digestion site): FIE I forward: 5' GCATTGCAATCCTATGCTGA 3' and FIE I reverse: $5^{\prime}$ TGTGGGGAATTTTGATGGAT 3' (Genomed, Poland). Primers for FIE (one digestion site): FIE III forward: 5' GCTTGTGGTTCGTTTGTATG 3' and FIE III reverse: 5'CCTATATGGCAACAGAAAAT 3' (Genomed, Poland). A protocol was used for PCR amplification: the initial denaturation round $\left(95^{\circ} \mathrm{C}\right.$ for $\left.5 \mathrm{~min}\right)$ was followed by thirty cycles of $95^{\circ} \mathrm{C}$ for $30 \mathrm{~s}, 58^{\circ} \mathrm{C}$ for $30 \mathrm{~s}$, and $72^{\circ} \mathrm{C}$ for $50 \mathrm{~s}$. The final round used $72^{\circ} \mathrm{C}$ for $5 \mathrm{~min}$.

\section{Results}

\section{Stage of female gametophyte development in unfertilized ovules at inoculation}

In unfertilized ovules different stages of female gametophytes (FGs) were observed starting from 4-nucleate to mature 7-celled female gametophyte. The mature FG consisted of an egg apparatus on micropylar pole, antipodals at chalazal pole and central cell with 2 polar nuclei or secondary nucleus formed after fusion of polar nuclei similarly as previously described by Rojek et al. [10]. In analyzed ovules, mature female gametophytes occurred with highest frequency $(\sim 80 \%)$, confirming that the mature FG was the best stage for autonomous endosperm induction. Embryos and endosperm were not observed in analyzed ovules.

\section{Vitality of pistils and ovules during in vitro culture}

Pistils cultured on the media with the addition of steroid hormones were conspicuously enlarged on the 7th day when compared to the size of pistils at inoculation (Fig. 1). Also, their viability was high reaching more than $84 \%$ (Appendix S1).

Steroid hormones added to the culture media promoted growth and intensive proliferation of pistil tissues in subsequent days of culture. Ovaries, despite the significant overgrowth of the lower part, immersed in the medium, were green and viable (Fig. 1e,f). Proliferation of the ovaries was accompanied by enlargement of the ovules. On the 7 th day of culture, the most enlarged ovules were located in the lower part of the ovary. The increase in ovule size resulted in a decrease in the number of ovules, as compared to ovules number at inoculation ( $50 \pm 0.19$ ovules per ovary). The average number of ovules inside each ovary was between 28 and 35 at 7 th day of culture (Tab. 1).

\section{Female gametophyte and autonomous endosperm development}

During in vitro culture with estrone (M2), androsterone (M3), progesterone (M4) and epiBL (M5) both FGs and autonomous endosperm development were noted (Fig. 2Fig. 6).
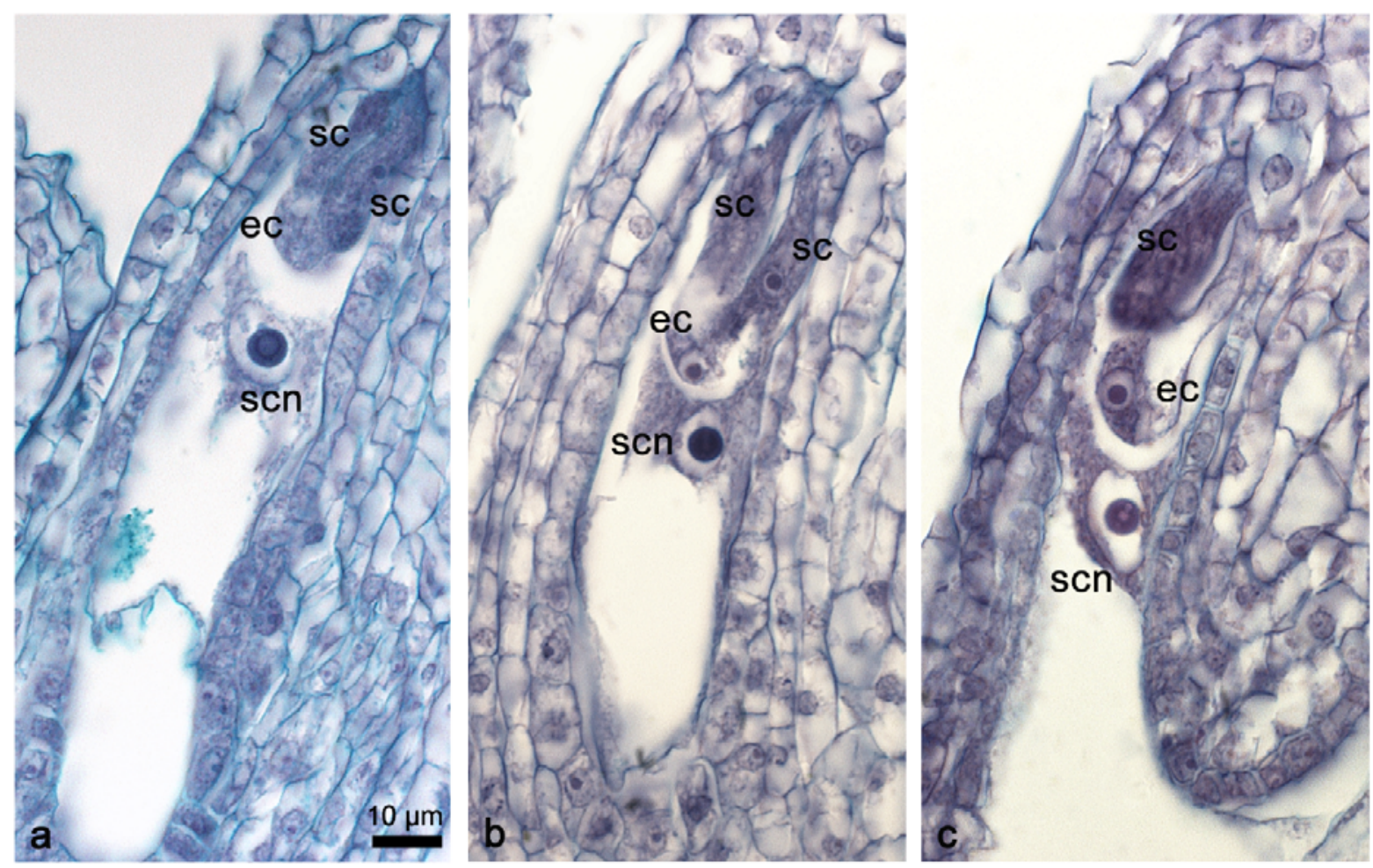

Fig. 2 Female gametophytes at 7th day of in vitro culture. Longitudinal paraffin sections of Arabidopsis thaliana Col-0 ovules inside unpollinated ovaries, cultured in vitro. A mature female gametophyte at 7th day on M2 (a), M3 (b) and M5 (c); an egg cell $(\mathrm{ec})$, synergids (sc) and secondary central cell nucleus $(\mathrm{scn})$ are visible. Scale bar at a fits also for other figures. 

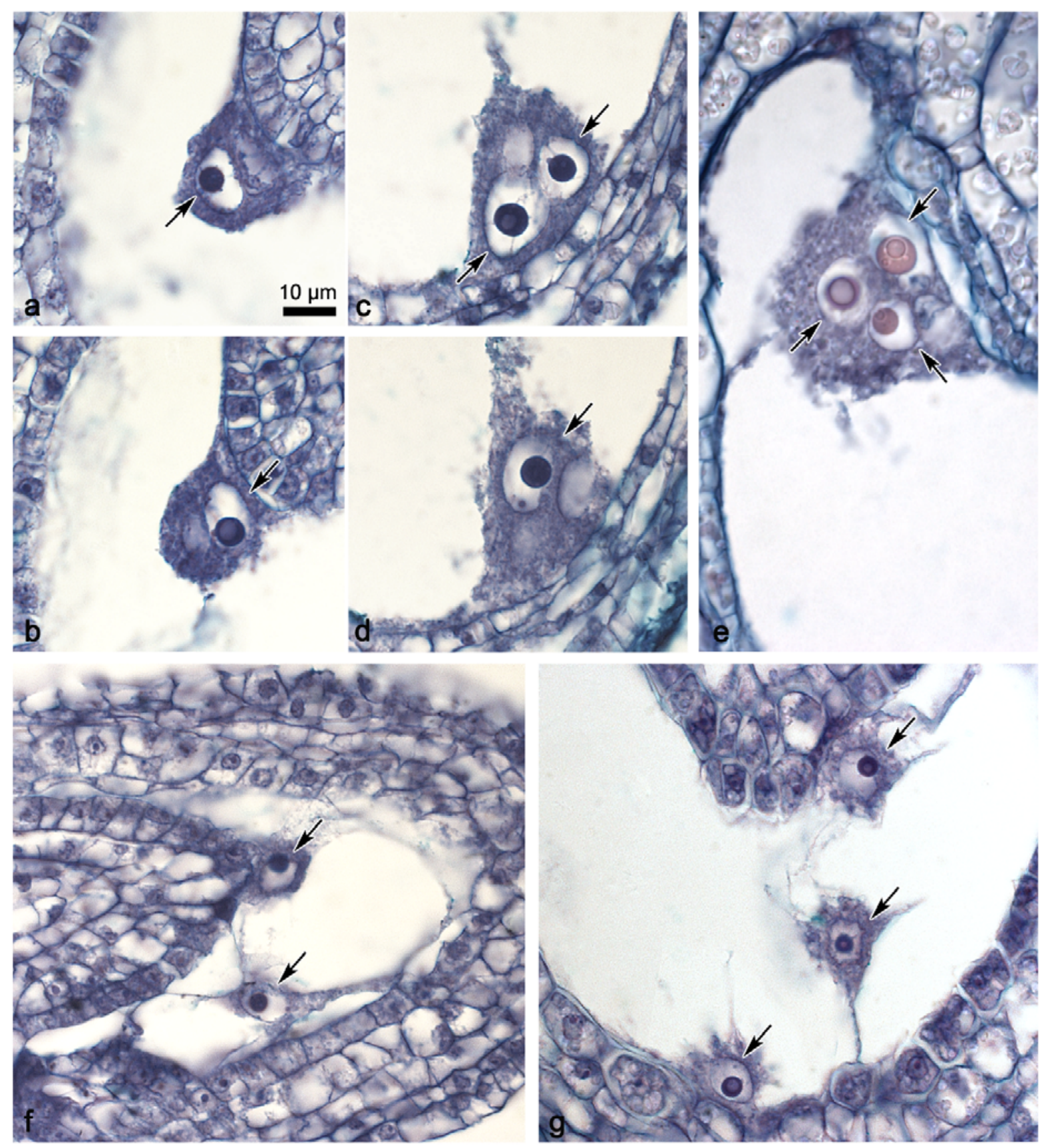

Fig. 3 Few-nuclear autonomous endosperm (AE1) induced in vitro. Longitudinal paraffin sections of Arabidopsis thaliana Col-0 $(\mathbf{a}-\mathbf{d}, \mathbf{f}, \mathbf{g})$ and fie-1/FIE (e) ovules inside unpollinated ovaries, cultured in vitro on M2 (a-d), M3 (f), M4 (e) and M5 (g) for 7 days. The early stages of autonomous endosperm development. a,b 2-nucleate AE. Few-nucleate AE; big nuclei clustered in dense mass of cytoplasm $(\mathbf{c}, \mathbf{d})$ or in cytoplasmic strands $(\mathbf{f}, \mathbf{g})$. AE nuclei indicated by arrows. Scale bar at a fits also for other figures.

In ovules inside 7-day-old ovaries, still viable FGs were observed containing central cell with secondary nucleus and intact egg apparatus (Fig. $2 \mathrm{a}-\mathrm{c}$ ) or sporadically two polar nuclei in central cell.

Autonomous endosperm was observed in ovules cultured on all steroid hormone-enriched media, in two study genotypes, with the expected higher AE frequency in fie-1 genotype, due to the presence of mutation (Fig. 7). As the autonomous endosperm showed various degrees of advancement in the development, it was divided into four developmental stages: (i) AE few-nuclear (AE1); (ii) AE multinuclear
(AE2); (iii) AE multinuclear just before cellularization (AE3) and (iv) AE cellular (AE4; Tab. 2, Fig. 8, Fig. 9).

Few-nuclear AE (AE1) consisted of 2-10 nuclei, very often close to each other clustered in the mass of cytoplasm in the middle part of FG (Fig. 3c,d) or near the wall, where the FG banded and created a characteristic horseshoe shape (Fig. 3a,b,e). Sometimes the regular arrangement of AE nuclei in the whole FG was noted (Fig. 3f,g). The first category of AE was dominant on control (M1) medium for both genotypes and on M2 medium for wild-type (Tab. 2, Fig. 8 and Fig. 9). 

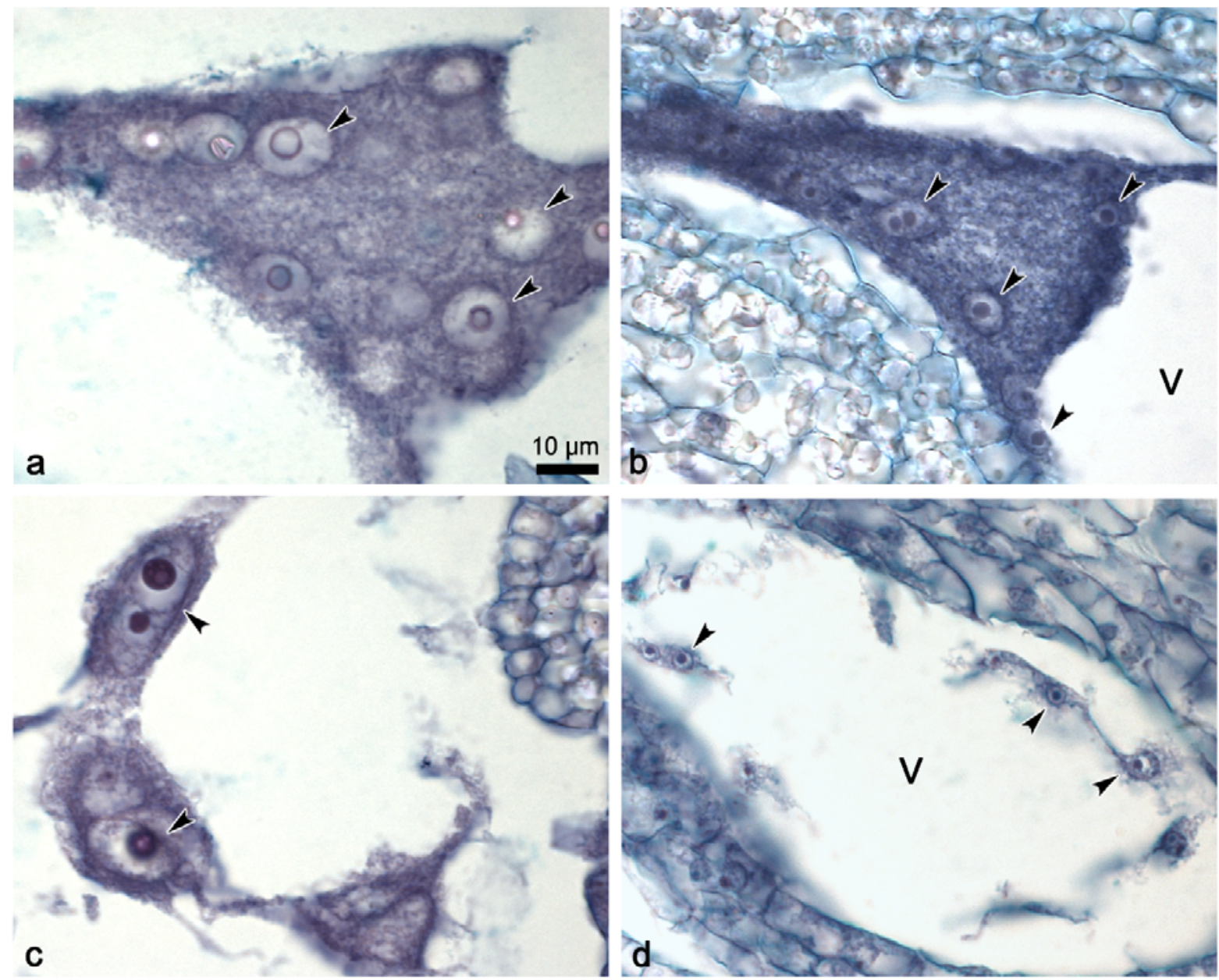

Fig. 4 Multinuclear autonomous endosperm (AE2) induced in vitro. Longitudinal paraffin sections of Arabidopsis thaliana Col-0 (d) and fie-1/FIE (a-c) ovules inside unpollinated ovaries, cultured in vitro on M2 (d), M3 (b) and M4 (a,c) media, for 7 days. a,b Multinuclear stage of AE with characteristic feature for fie-1 phenotype, at micropylar pole of FG; differences in size of $\mathrm{AE}$ nuclei are visible. c AE nuclei grouped into 2 or 3 nuclei and surrounded by dense cytoplasm. d Small AE nuclei positioned around central vacuole. AE nuclei were indicated by arrows; v - vacuole. Scale bar at a fits also for other figures.

Multinuclear autonomous endosperm (AE2) consisted of numerous nuclei dipped in cytoplasm of the central cell. The size and distribution of AE nuclei in FGs were different. AE nuclei were positioned in cytoplasmic strands or clustered in big one multinuclear mass (Fig. 4a) or few-nuclear units of dense cytoplasm (Fig. 4c). Overproliferation of AE2 at micropylar and chalazal poles of FG was characteristic of fie-1/FIE genotype (Fig. 4b). Many of ovules of Col-0 possessed few-nuclear clusters or single nuclei, which were arranged around central vacuole (Fig. 4d).

Multinuclear AE just before cellularization (AE3) showed characteristic features, which resembled fertilization-derived multinuclear endosperm just before cellularization. AE nuclei arranged around central vacuole, parietal and surrounded by cytoplasm forming structures resembling nuclear cytoplasmic domains. AE nuclei with cytoplasmic strands looked like stars network (Fig. $5 \mathrm{a}-\mathrm{c}$ ) or like a spider web with nuclei anchored (Fig. 5d).

The most advanced stage of AE development - cellular (AE4) - resembled the tissue which fills the embryo sac (Fig. 6a-e). The cytochemical staining of Alcian blue indicated the presence of walls between AE nuclei. AE tissue was clearly separated from other tissues of the ovules by integumentary tapeum. Sometimes the autonomous endosperm was only partially cellular (Fig. 6e).

The intact egg cell and synergids (one or two) accompanied AE in many ovules up to AE3 stage. An increased egg cell was present mostly on medium with progesterone (M4). In many ovules, the space separating the egg apparatus from the endosperm was very clear. At the AE4 stage, cellular endosperm was mostly accompanied by degenerated egg apparatus, which was still clearly separated from the endosperm space (Fig. 6e).

\section{The frequency of autonomous endosperm}

Autonomous endosperm was induced on all the used media and in each of the three samples within both genotypes. Statistical analysis revealed that the genotype type was especially important for the relative frequency of the occurrence of AE on various media types $\left(F_{4,16}=202.14\right.$, $P<0.001$; Appendix S2). As expected, genotype fie-1/FIE, showed a very high percentage of ovules with $\mathrm{AE}$ (between 

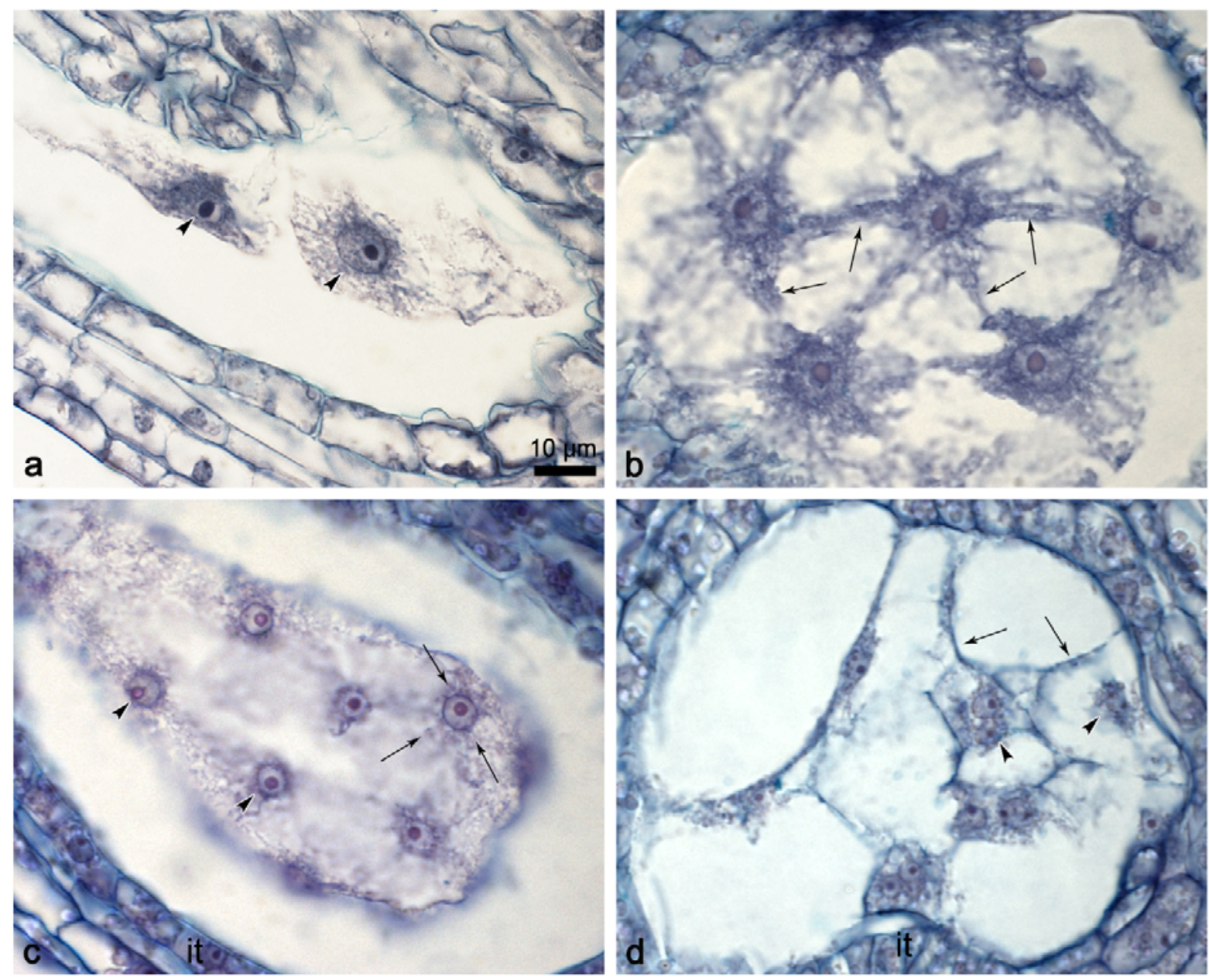

Fig. 5 Multinuclear AE just before cellularization (AE3) induced in vitro. Longitudinal paraffin sections of Arabidopsis thaliana fie-1/FIE ovules inside unpollinated ovaries, cultured in vitro on M3 (a,b) and M4 (c,d) media, for 7 days. a-c AE nuclei arranged regular and surrounded by "star-like" cytoplasmic network (arrows). d AE nuclei with cytoplasmic strands (arrows) looked like a spider web with nuclei anchored. AE nuclei were indicated by arrows; it - integument. Scale bar at a fits also for other figures.

$27.4 \%$ and $46.9 \%)$ compared to the wild genotype Col-0 (0.9-2.0\%; Fig. 7). Despite the drastic difference in the overall appearance of the AE frequency between genotypes, similar and significant differences in frequency for each media have been shown. The highest percentage of AE was induced on media M4 and M5 for both genotypes, and the lowest in the control medium without exogenous steroid hormones - M1. In the case of Col-0, the difference in the percentage of $\mathrm{AE}$ induction between media was small but in most cases statistically significant, at $P<0.05$ level (Fig. 7, Appendix S2). The exception was the similar percentage of AE induction for the M1 and M3 media ( $0.9 \%$ and $1.1 \%$, respectively; Tab. 1, Fig. 7 and $P=0.835$ for HSD post-hoc test; Appendix S2) and also for the M4 and M5 media (1.6\% and $2.0 \%$, respectively; $P=0.173$ for HSD post-hoc test). For fie-1/FIE genotype, differences between media were much clearer: for media M4 and M5, AE induction was over $10 \%$ higher than on the M1-M3 media. Moreover, statistical analysis revealed the significance of the observed differences, except media M1 and M2 ( $P=0.963$ for HSD post-hoc test).

\section{The development of AE in different treatments}

The relative proportions of the occurrence of subsequent AE developmental stages were analyzed within two studied genotypes. The analysis of variance showed a significant impact of used media (M1-M5) on the degree of advancement in the development of AE (four stages AE1-AE4; $F_{12,32}$ $=52.79, P<0.001$ for Col- 0 and $F_{12,32}=520.71, P<0.001$ for fie-1/FIE; Appendix S3, Appendix S4).

In wild-type Col-0, the youngest stage of AE development (AE1) was the only one found on M1 (0.9\%) and was the dominant type of developmental stages on M2 (0.7\%; Tab. 2, Fig. 8). Also, post-hoc test exhibited no differences between the two aforementioned media, for AE1 stage $(P=0.897$ for HSD post-hoc test; Appendix S3). Multinuclear stages of endosperm (AE2 and AE3) were presented on the M2-M5 media, but similar and significantly higher relative proportions of AE2 stage were observed on M4 and M5 media ( $0.7 \%$ for the M4 and $0.8 \%$ for M5), which was also confirmed by HSD post-hoc test $(P=1.000)$. 

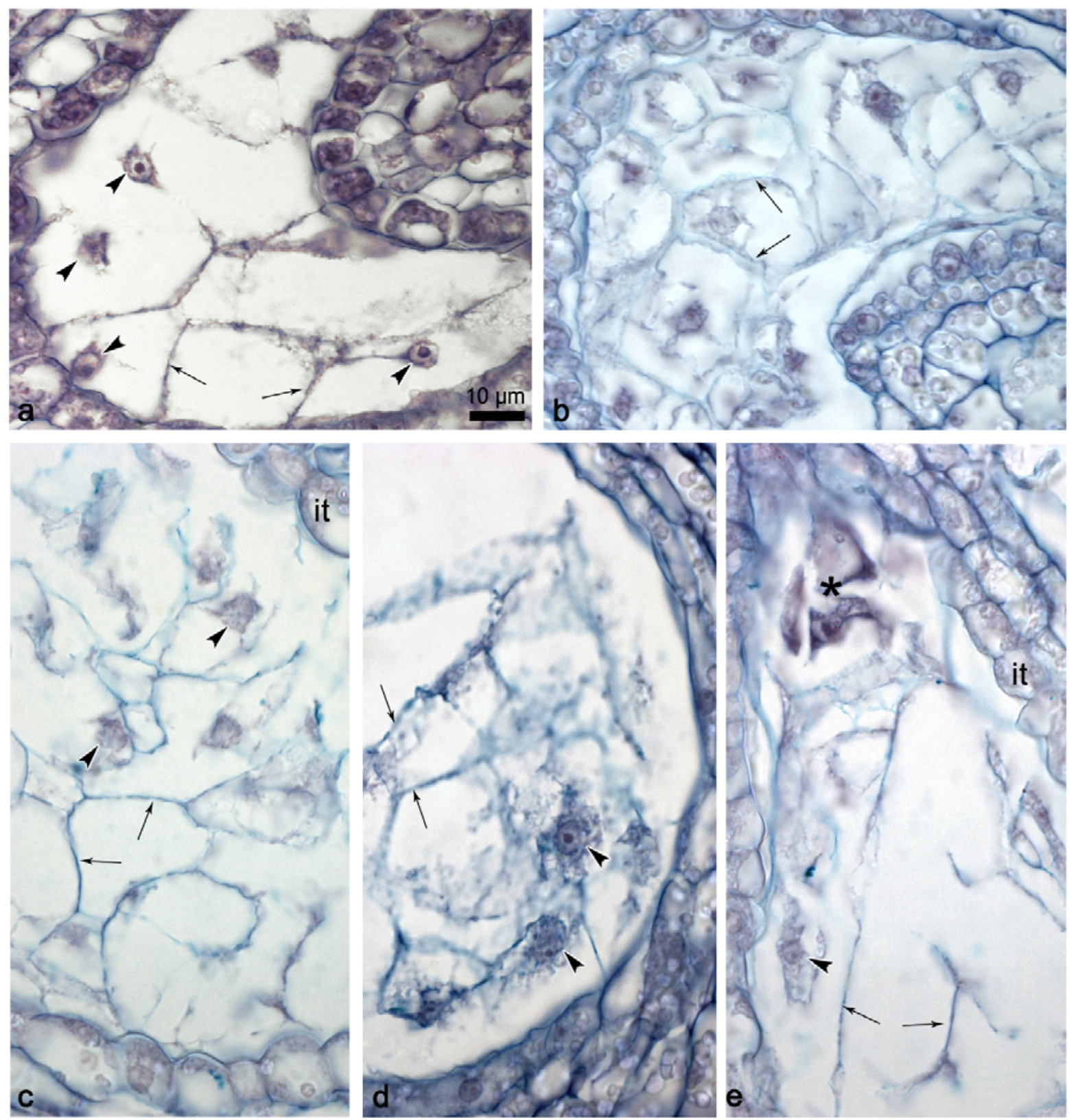

Fig. 6 Cellular autonomous endosperm (AE4) induced in vitro. Longitudinal paraffin sections of Arabidopsis thaliana fie-1/ FIE ovules inside unpollinated ovaries, cultured in vitro on M2 (c,e), M4 (d) and M5 (a,b) media, for 7 days. a-e Cellular stage of AE. The AE nuclei (arrowheads) separated by alcian blue-stained partitions (arrows). e Partly cellular AE accompanied by degenerated egg apparatus (star). it - integument. Scale bar at a fits also for other figures.

Cellular stage (AE4) was presented only on the M4 and M5 media, with higher proportion at M5 (0.3\% for M4 and $0.6 \%$ for M5; Tab. 2, Fig. 8), but still similar to relative proportion as on M4 $(P=0.341$ for HSD post-hoc test).

The different relationships were received for the mutant genotype (fie-1/FIE) on the studied media, but were similar to the Col- 0 wild-type. The most differentiating values showed the M4 and M5 media $(P<0.001$ for HSD post-hoc test; Appendix S4). Because of the described mutation, there no statistically significant differences in the high-frequency stage AE2 for the M1-M5 media (from M4-14.0\% to M5-18.6\%; Tab. 1, Fig. 9), as multinuclear, undifferentiated autonomous endosperm is characteristic of unfertilized fie- 1 ovules. The appearance of the remaining AE stages was extremely important. The youngest stage AE1 was observed very often on M5 medium (14.1\%) and also was represented on M1 (7.4\%), but their relative proportions were statistically different $(P<0.001$ for HSD post-hoc test). The frequency of AE3 stage was highly dependent on the used medium: the least frequently on M1 (1.1\%) and the most commonly on M4 (19.8\%; Tab. 2, Fig. 9, Appendix S4). AE4 stage was not present on the control medium - M1. However, in other cases, frequency of AE4 was the lowest among all the developmental stages of $\mathrm{AE}$, with the highest percentage on M4 (4.8\%). In fie-1/FIE heterozygote, advanced stages of $\mathrm{AE}$ development (AE3 and AE4) emerged with the greatest 


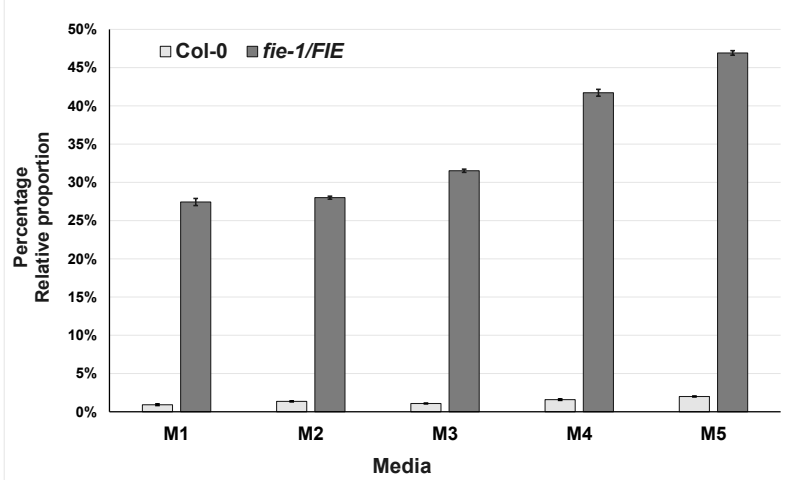

Fig. 7 Quantification of the total induction of autonomous endosperm in Col-0 and fie-1/FIE genotypes after 7 days of in vitro culture on M1-M5 media. Each column of the histogram represents the percentage of ovules with AE. Bars indicate $S E_{m}$. Statistical analysis revealed that the type of genotype was especially important for the relative frequency of the occurrence of $\mathrm{AE}$ on various media types $\left(F_{4,16}=202.14, P<0.001\right)$. As expected, genotype fie-1/FIE, showed a very high percentage of ovules with $\mathrm{AE}$ compared to the wild genotype Col-0. Despite the drastic difference in the overall appearance of the AE frequency between genotypes, similar and significant differences in frequency for each media have been shown. The highest percentage of AE was induced on media M4 and M5 for both genotypes, and the lowest in the control medium without exogenous steroid hormones - M1. In the case of Col-0, the difference in the percentage of $\mathrm{AE}$ induction between media was small but in most cases statistically significant, at $P<0.05$ level.

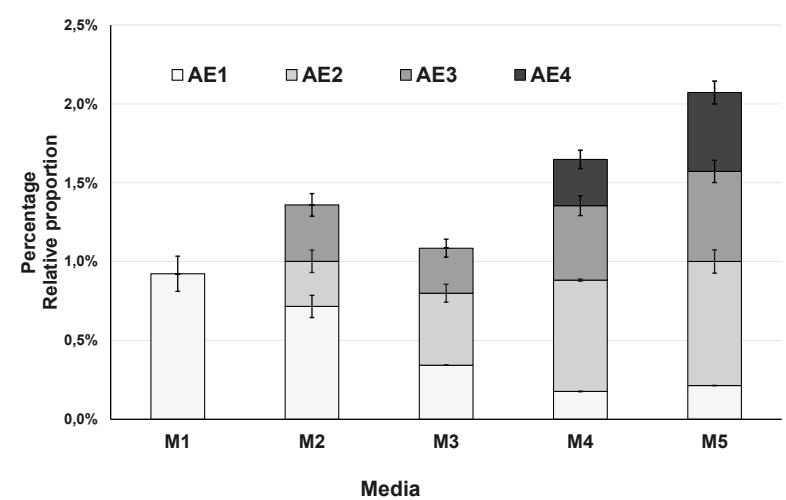

Fig. 8 Quantification of the occurrence of four developmental stages of autonomous endosperm development in Col-0, after 7 days of in vitro culture on M1-M5 media. Each column of the histogram represents the percentage of ovules that showed particular developmental stages of autonomous endosperm (AE1-AE4). Bars indicate $S E_{\mathrm{m}}$. In wild-type Col-0, the youngest stage of $\mathrm{AE}$ development (AE1) was the only one found on M1 and was the dominant type of developmental stages on M2. Multinuclear stages of endosperm (AE2 and AE3) were present on the M2-M5 media, but similar and significantly higher relative proportions of AE2 stage were observed on M4 and M5 media ( $0.7 \%$ for the M4 and $0.8 \%$ for M5). Cellular stage (AE4) was present only on the M4 and M5 media, with higher proportion at M5 (0.3\% for M4 and 0.6\% for M5), but still similar to relative proportion as on M4 ( $P=0.341$ for HSD post-hoc test).

Tab. 2 The occurrence of four developmental stages of autonomous endosperm (AE) in unpollinated ovules of Arabidopsis thaliana on media with steroid hormones and on hormone-free medium (M1 - control).

\begin{tabular}{|c|c|c|c|c|c|c|c|c|c|}
\hline \multirow{2}{*}{$\begin{array}{r}\text { Medium } \\
\text { Col-0 }\end{array}$} & \multirow{2}{*}{$\begin{array}{c}\text { No. of } \\
\text { analyzed } \\
\text { viable } \\
\text { ovules at } \\
\text { 7th day of } \\
\text { culture }\end{array}$} & \multicolumn{8}{|c|}{ Percentage (\%) of ovules with AE developmental stages and accompanied structures ${ }^{1}$} \\
\hline & & \multicolumn{2}{|c|}{ AE1;2-10n } & \multicolumn{2}{|c|}{ AE2; multinuclear } & \multicolumn{2}{|c|}{$\begin{array}{c}\text { AE3; before } \\
\text { cellularization }\end{array}$} & \multicolumn{2}{|c|}{ AE4; cellular } \\
\hline M1 & 866 & 8 & $(0.9)$ & 0 & $(0.0)$ & 0 & $(0.0)$ & 0 & $(0.0)$ \\
\hline M2 & 1397 & $5+5^{1}$ & $(0.7)$ & 4 & $(0.3)$ & 5 & $(0.4)$ & 0 & $(0.0)$ \\
\hline M3 & 1751 & 6 & $(0.3)$ & 8 & $(0.5)$ & 5 & $(0.3)$ & 0 & $(0.0)$ \\
\hline M4 & 1700 & 2 & $(0.2)$ & $8+4^{1}$ & $(0.7)$ & $5+3^{1}$ & $(0.5)$ & 5 & $(0.3)$ \\
\hline M5 & 1400 & 2 & $(0.2)$ & $8+3^{1}$ & $(0.8)$ & $6+2^{1}$ & $(0.6)$ & 7 & $(0.5)$ \\
\hline \multicolumn{10}{|c|}{$f i e-1 / F I E$} \\
\hline M1 & 908 & $50+17^{1}$ & (7.4) & $151+10^{1}$ & $(17.7)$ & 10 & (1.1) & 0 & $(0.0)$ \\
\hline M2 & 1386 & $18+5^{1}$ & (1.7) & $243+9^{1}$ & $(18.2)$ & $75+20^{1}$ & (6.9) & 17 & (1.2) \\
\hline M3 & 1593 & 8 & $(0.5)$ & $253+7^{1}$ & $(16.3)$ & 194 & $(12.2)$ & 40 & (2.5) \\
\hline M4 & 1700 & 54 & $(3.2)$ & $231+7^{1}$ & $(14.0)$ & $316+20^{1}$ & $(19.8)$ & $80+1^{1}$ & (4.8) \\
\hline M5 & 1396 & $184+13^{1}$ & $(14.1)$ & $249+11^{1}$ & $(18.6)$ & $185+5^{1}$ & (13.6) & 8 & (0.6) \\
\hline
\end{tabular}

${ }^{1}$ No. of ovules with AE accompanied by intact egg cell; $\mathrm{n}$ - nuclei. 


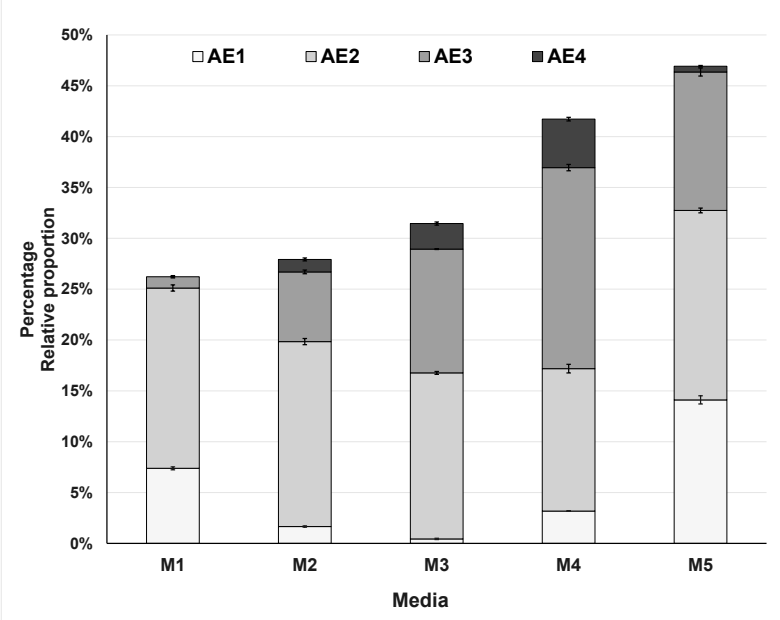

Fig. 9 Quantification of the occurrence of four developmental stages of autonomous endosperm development in fie-1/FIE, after 7 days of in vitro culture on M1-M5 media. Each column of the histogram represents the percentage of ovules that showed particular developmental stages of autonomous endosperm (AE1-AE4). Bars indicate $S E_{m}$. The high-frequency of AE2 stage was the result of a mutation. The appearance of the remaining AE stages was extremely important. The most differentiating values showed the M4 and M5 media $(P<0.001$ for HSD post-hoc test; Appendix S3). Because of the described mutation, there no statistically significant differences in the high-frequency stage AE2 for the M1-M5 media, as multinuclear, undifferentiated autonomous endosperm is characteristic of unfertilized fie-1 ovules. The appearance of the remaining AE stages was extremely important. The youngest stage AE1 was observed very often on M5 medium (14.1\%) and also was represented on M1 (7.4\%). The frequency of AE3 stage was highly dependent on the used medium: the least frequently on M1 (1.1\%) and the most commonly on M4 (19.8\%). AE4 stage was not present on the control medium - M1. The frequency of AE4 was the highest percentage on M4 (4.8\%). In fie-1/FIE heterozygote, advanced stages of AE development (AE3 and AE4) emerged with the greatest frequency on M4.

frequency on M4, in contrast to the wild-type Col-0, where high frequencies of advanced stages of AE on M4 and M5 media were observed (Fig. 8).

\section{Methylation analysis in FIE gene}

In this study we also established how stress in vitro affects methylation of FIE gene - one of the core genes, which product, together with other of PRC2 components act and maintain the paternally expressed genes to be silent [36]. Using standard media (with auxin and cytokinin) and 5-azacytidine for culture of unfertilized ovules, we expected to obtain primary indication for further molecular investigation.

To analyze methylation in FIE gene we used standard media for Col-0 unpollinated ovaries culture: M1 medium, M6 medium with balanced concentration of auxin and cytokinin (MS + suc6\% + $0.1 \mathrm{mg} \mathrm{l}^{-1} \mathrm{NAA}+2 \mathrm{mg} \mathrm{l}^{-1} \mathrm{BAP}$; see [10]), and M7 medium with demethylating factor (MS + suc6\% $+50 \mu \mathrm{M} 5$-azaC). Analysis of the methylation using the restriction method combined with PCR has shown differences in the level of methylation of explants cultured on different media. Based on band intensity after PCR for a specific primer pairs (without cutting and after cutting of enzymes) of FIE gene, the differences in the methylation were preliminary (qualitatively) determined (Fig. 10a,b). Changes in the level of methylation of FIE gene have occurred under in vitro conditions. The influence of a particular medium on the degree of analyzed gene methylation was similar, regardless of the type of explant (ovaries or seedlings). At the 7th day of in vitro culture, the methylation level seemed to be increased on M1 medium without hormones (Fig. 10a; strong band No. 2 visible on the gel) but decreased (weaker bands) on M6 medium, which was supplemented with auxin and cytokinin (sample No. 3). M7 medium with 5-azaC generally gave weaker (for ovaries) and very weak (for seedlings) bands in the gel (samples No. 4 and No. 6; Fig. 10a), suggesting decreased DNA methylation. An ambiguous band image could be the result of the residue in the reaction mixture of non-cut copies of FIE. Different (often opposite) intensity of bands for HpaII and MspI (Fig. 10a; sample No. 3; band intensity was lower for the HpaII and greater for MspI) indicated the specificity of methylation sites (methylated/demethylated is the most outer cytosine in the CCGG sequence), and nonuniform degree of methylation of both DNA strands (DNA hemimethylation [34]).

\section{Discussion}

The present studies are a continuation of our long-term research on overcoming the fertilization requirement and inducing the divisions of unfertilized central cell in sexually reproduced taxa from different genera and families, including a model plant Arabidopsis thaliana (wild genotypes Col-0, MET1/MET1, FIE/FIE and met 1 and fie-1 mutants [10]). As a universal technique, we preferred the culture of unpollinated pistils and stimulation by phytohormones [41]. Previously, a combination of auxins and cytokinins was the best for fertilization-free endosperm development [10]. Based on Arabidopsis experiments, we hypothesized that Arabidopsis represents a good model to investigate the phenomenon of apomictic model of endosperm development and that steroid hormones, especially mammalian sex hormones are promising [42]. In fact, stimulation of secondary nucleus of female gametophyte to division and further development of AE were achieved for both kind of hormones, MSHs and brassinosteroid.

\section{Autonomous endosperm in vitro can fully develop \\ through steroid hormones stimulation}

In Col-0 genotype, the highest frequency of ovaries/ ovules with AE ( $60 \%$ ovaries / $6 \%$ ovules) was noted on media with combinations of auxin and cytokinin [10] and supplied with $50 \mu \mathrm{M} 5$-azacytidine (M7 medium, 38\%/2\% of the ovaries/ovules; Appendix S5, Fig. S1). Although, neither epibrassinolide nor mammalian sex hormones increased $\mathrm{AE}$ frequency in unpollinated ovules of Col-0, they evidently improved the frequency of $\mathrm{AE}$ occurring in fie-1 mutant ovules, as was compared with frequency on P1-P3 media, 


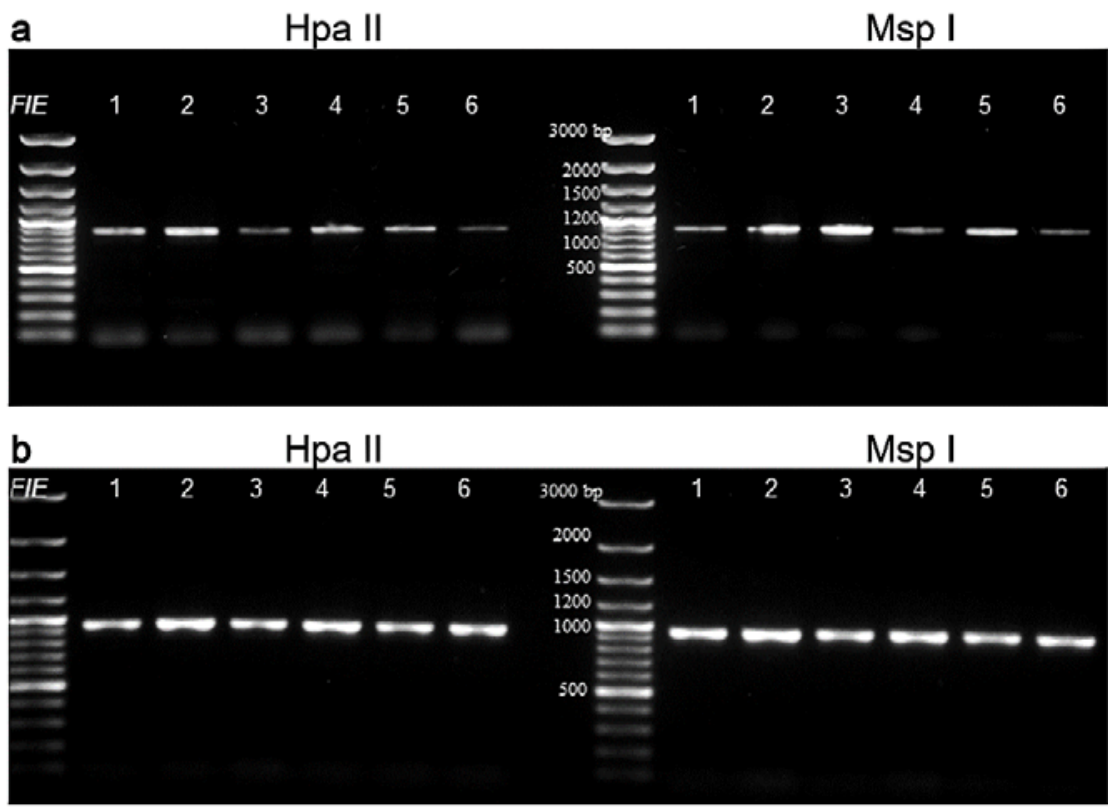

Fig. 10 Analysis of DNA methylation in FIE gene. Changes in DNA methylation in FIE gene of Col-0 seedlings and ovaries analyzed at inoculation ( 0 day of culture) and after 7 days of in vitro culture. a PCR analysis for FIE gene; lines 1-6: investigated individual trials/experiments; other lines: GeneRuler 100bp plus. HpaII/MspI isoschizomers (sensitive to methylation) were used to digestion of genomic DNA in FIE. b The positive control for FIE - the lack of digestion sites for HpaII/MspI isoschizomers (undigested DNA). Descriptions of the bands: 1 - ovaries at inoculation; 2 - ovaries cultured on M1 (MS + 6\% sucrose); 3 - ovaries cultured on M6 (MS $+6 \%$ sucrose $\left.+0.1 \mathrm{mg} \mathrm{l}^{-1} \mathrm{NAA}+2 \mathrm{mg} \mathrm{l}^{-1} \mathrm{BAP}\right) ; 4$ - ovaries cultured on M7 with 5 -azaC (MS $+6 \%$ sucrose $+50 \mu \mathrm{M}$ 5-azaC); 5 - seedlings cultured on M1; 6 - seedlings cultured on M7. The fluorescent intensity of investigated bands indicate the degree of DNA methylation. The influence of a particular medium on the degree of analyzed gene methylation was similar, regardless of the type of explant (ovaries or seedlings). a DNA methylation in FIE changes in vitro; these changes are different for individuals media (line 2-6). The methylation level of FIE seemed to be increased on M1 medium (a; strong band No. 2 visible on the gel) without growth regulator, in comparison to inoculation stage (a; band No. 1), but decreased (weaker bands) on M6 medium, which was supplemented with auxin and cytokinin (a; sample No. 3). M7 medium with 5 -azaC generally gave weaker (for ovaries) and very weak (for seedlings) bands in the gel (a; samples No. 4 and No. 6), suggesting decreased DNA methylation. An ambiguous band image could be the result of the residue in the reaction mixture of non-cut copies of FIE.

as noted by Rojek et al. [10]. This is certainly related to the higher ovules survival in vitro culture. Also, there is no doubt that the steroid hormones stimulate the viability of developing ovules and further AE development till cellular stage. According to our knowledge, we are the first to use steroid hormones to stimulate female gametophyte development and to induce AE formation (see also [42]), although brassinosteroids are widely applied in the field of in vitro cultures [20]. Application of MSHs resulted in stimulation of development but only in the presence of certain concentration $[43,44]$. To determine the most favorable dose of particular MSH for the AE induction and development, other concentrations of hormones should be investigated.

Similar to our previous studies on unfertilized ovules of Arabidopsis in cultures, AE development was delayed as compared to findings in planta $[8,10]$ but the successive stages from 2-nucleate to cellular AE were observed in vitro. The pattern of in vitro-induced AE development was similar to that of endosperm of fis mutant in planta [7] and also to $\mathrm{AE}$ produced in unfertilized ovules of met 1 mutants in vitro [10]. AE originated from the secondary nucleus and at binucleate stage two adjacent nuclei were located near the egg cell or in the place where the embryo sac is bent similar as in endosperm development in planta after fertilization [45]. The autonomous origin of AE strongly support the presence of intact egg cell and/or synergid(s) in the same ovule. Moreover, the appearance of the ovules containing $\mathrm{AE}$ at a very early developmental stage in fie-1 mutant (AE1 stage, which was very younger than guaranteed by the mutation) indicates induction of endosperm development, which was independent of fertilization and mutation. The most important consequence of the action of media with steroid hormones was the advancement of endosperm development much further and achieving maturity (AE4 stage - cellular). The overthrow of the general phenomenon occurring in fis mutants [1] - the lack of cellularization of autonomous endosperm - gives hope for finding the key which allows switching the AE development from nuclear to cellular. 
All tested hormones in the present study and in previous experiments induced central cell division and AE development in unfertilized ovules of Arabidopsis genotypes [8-10]. They did not stimulate divisions of egg cell and haploid embryo formation although embryo-like structures of unknown origin were observed on medium with epiBL and in fie-1/FIE Arabidopsis mutant cultured on M1 (data not shown). The proper and full development of embryo is strongly dependent on the cellularization stage of endosperm and sucrose influx from the endosperm [1]. It is possible that higher concentration of sucrose in vitro (6\%) and steroid hormones are sufficient to induce full endosperm development, but not yet to develop a parthenogenetic embryo. To induce unfertilized egg cell development, steroid hormones other than brassinosteroids have to be used because the embryo development is brassinosteroid independent [46].

\section{Changes in DNA methylation as a probable indicator of apomictic-like development in Arabidopsis in vitro}

Analysis of FIE gene showed differences in the level of FIE methylation in Col-0 explants cultured on different media. At the moment of inoculation the DNA methylation in genes was relatively low. Also, analysis of histone H3K9 methylation showed low level of methylation prior to fertilization (Appendix S6, Fig. S2). Hormone-free medium, but with a higher concentration of sugar, seems to affect the greatest increase of methylation in the gene. However, the effect of medium with 5 -azaC is highly demethylating, provided that 5 -azaC has been incorporated into DNA during replication. Therefore, global methylation after 5-azaC treatment was significantly lower in seedlings, where the number of cell divisions is commonly known to be very high [34,47], in contrast to the mature ovaries and ovules (rare cell divisions in somatic tissues and in female gametophytes).

The preliminary results indicate changes in methylation in individual sites cuts of HpaII/MspI enzymes. If we considered the general trend of reduction in the level of methylation in the tissues cultured in vitro, the FIE gene would exhibit relatively high activity in ovary tissues. In vivo,

\section{Acknowledgments}

We thank Prof. Nir Ohad (Tel Aviv University, Israel) and their colleagues for providing fie-1/FIE seeds, primer sequences and valuable assistance. Special thanks go to Prof. Andrzej Wierzbicki for providing method of preliminary DNA methylation analysis, helpful discussion and critical comments on the manuscript. We thank Prof. Anna Janeczko for providing methods of steroid hormones using and valuable assistance, and Dr. Roman Synak for help in statistical analyses. This work was partly founded from Polish Ministry of Science and Higher Education (project N30300731/0281) and University of Gdańsk (projects 1412-50387-8; L160-5-0415-0).

\section{Authors' contributions}

The following declarations about authors' contributions to the research have been made: study conception and design: JR; acquisition of data: JR, ŁP, $\mathrm{MK}$; analysis and interpretation of data: JR, AN, ŁP; drafting of manuscript: JR, AN; critical revision of the manuscript: JB.

\section{Competing interests}

No competing interests have been declared. the FIE activity is high before fertilization and just after fertilization [48]. Perhaps regulation of its expression occurs, as in the case of $M E A$, by antagonistically acting proteins, viz., FIS-PCR2 (for H3K27me3 methylation), methyltrasferase MET1(for DNA methylation) and demethylase DME [6]. Thus, the low level of methylation FIE of both in vitro and in vivo may indicate the function of the similarity in the initiation and development of endosperm after fertilization and endosperm formed independently. The effect of significant enhancement of AE development in Col-0 and fie-1/FIE induced by steroid hormones applied in vitro could be synergistic effect of changes in histone modification and DNA methylation within distinct set of common target genes involved in autonomous endosperm development, as was hypothesized for met1-3/MET1; mea-1/MEA [6]. Therefore, the methylation analysis of FIE and MEA in fie-1/ FIE genotype is an urgent task.

\section{Conclusions}

Our studies on the initiation of apomictic processes have focused on the model plant Arabidopsis thaliana, which belongs to the same family as sexual Brassica napus and apomictic species of Boechera genus. Stimulation of the central cell to fertilization-free endosperm development induced by the application of different steroid hormones in culture of unfertilized ovules confirmed the ability of Arabidopsis thaliana (Col-0) to switch from sexual to apomictic endosperm development. The effect of steroid hormones should be further investigated at the cell level with the use of labeled mammalian steroids to visualize their accumulation site in ovule tissues. We also demonstrated that changes in environmental conditions (in vitro) are sufficient to initiate the steps of apomixis (production of autonomous endosperm), at least in Arabidopsis thaliana. The obtained results should enrich the knowledge of the mechanisms controlling seed development in Angiospermae and can be used for further work on introgression of the apomictic mechanisms to the reproduction of crop species.

\section{Supplementary material}

The following supplementary material for this article is available online at http://pbsociety.org.pl/journals/index.php/asbp/rt/suppFiles/ asbp.2015.022/0:

1. Appendix S1: viability of explants (unpollinated pistils) at 7 th day of in vitro culture.

2. Appendix S2: statistical analysis of AE induction.

3. Appendix S3: statistical analysis of the occurrence of AE developmental stages for Col-0.

4. Appendix S4: statistical analysis of the occurrence of AE developmental stages for fie-1/FIE.

5. Appendix S5: the description of autonomous endosperm development on M7 medium with 5-azacitidine.

6. Appendix S6: the method for immunodetection of H3K9.

7. Fig. S1: autonomous endosperm induced on M7 medium with 5-azacitidine.

8. Fig. S2: immunocytochemical detection of $\mathrm{H} 3$ histone methylation in control material. 


\section{References}

1. Lafon-Placette C, Köhler C. Embryo and endosperm, partners in seed development. Curr Opin Plant Biol. 2014;17:64-69. http://dx.doi. org/10.1016/j.pbi.2013.11.008

2. Tucker MR, Koltunow AMG. Sexual and asexual (apomictic) seed development in flowering plants: molecular, morphological and evolutionary relationships. Func Plant Biol. 2009;36:490-504. http:// dx.doi.org/10.1071/FP09078

3. Rodrigez-Leal D, Vielle-Calzada JP. Regulation of apomixis: learning from sexual experience. Curr Opin Plant Biol. 2012;15:549-555. http:// dx.doi.org/10.1016/j.pbi.2013.11.015

4. Ravi M, Marimuthu MPA, Siddiqi I. Gamete formation without meiosis in Arabidopsis. Nature. 2008;451:1121-1124. http://dx.doi. org/10.1038/nature06557

5. Eckardt A. A role for ARGONAUTE in apomixis. Plant Cell. 2011;23:430. http://dx.doi.org/10.1105/tpc.111.230211

6. Schmidt A, Wöhrmann HJP, Raissig MT, Arand J, Gheyselinck J, Gagliardini $\mathrm{V}$, et al. The Polycomb group protein MEDEA and the DNA methyltrasferase MET1 interact to repress autonomous endosperm development in Arabidopsis. Plant J. 2013;73:776-787. http://dx.doi. org/10.1111/tpj.12070

7. Vinkenoog R, Spielman M, Adams S, Fischer RL, Dickinson HG, Scott RJ. Hypomethylation promotes autonomous endosperm development and rescues postfertilization lethality in fie mutants. Plant Cell. 2000;12:2271-2282. http://dx.doi.org/10.1105/tpc.12.11.2271

8. Rojek J, Kuta E, Bohdanowicz J. In vitro culture promotes partial autonomous endosperm development in unfertilized ovules of wild-type Arabidopsis thaliana var. Columbia. Sex Plant Reprod. 2005;18:29-36. http://dx.doi.org/10.1007/s00497-005-0246-z

9. Kapusta M, Rojek J, Bohdanowicz J. Induction of autonomous endosperm development in ovules of unpollinated pistils of Arabidopsis thaliana var. Landsberg, cultured in vitro. Acta Biol Cracov Ser Bot. 2007;49:53-59.

10. Rojek J, Kuta E, Kapusta M, Ihnatowicz A, Bohdanowicz J. The influence of fie and met 1 mutation and in vitro culture conditions on autonomous endosperm development in unfertilized ovules of Arabidopsis thaliana. Acta Biol Cracov Ser Bot.2013;55:134-145. http://dx.doi.org/10.2478/abcsb-2013-0032

11. Bajguz A, Tretyn A. The chemical characteristic and distribution of brassinosteroids in plants. Phytochemistry. 2003;62:1027-1046. http:// dx.doi.org/10.1016/S0031-9422(02)00656-8

12. Janeczko A. Brassinosteroids in agriculture, horticulture and in vitro culture. Kosmos. 2005;2-3:259-265.

13. Yang CJ, Zhang C, Lu YN, Jin JQ, Wang XL. The mechanism of brassinosteroids' action: from signal transduction to plant development. Mol Plant. 2011;4:588-600. http://dx.doi.org/10.1093/mp/ssr020

14. Hewitt S, Hillman JR, Knights BA. Steroidal oestrogens and plant growth and development. New Phytol. 1980;85:329-350. http://dx.doi. org/10.1111/j.1469-8137.1980.tb03172.x

15. Kim SY, He Y, Jacob Y, Noh YS, Michaels S, Amasino R. Establishment of the vernalization-responsive, winter-annual habit in Arabidopsis requires a putative histone $\mathrm{H} 3$ methyl transferase. Plant Cell. 2005;17:3301-3310. http://dx.doi.org/10.1105/tpc.105.034645

16. Montoya T, Nomura T, Yokota T, Farrar K, Harrison K, Jones JGD, et al. Patterns of Dwarf expression and brassinosteroid accumulation in tomato reveal the importance of brassinosteroid synthesis during fruit development. Plant J. 2005;42:262-269. http://dx.doi. org/10.1111/j.1365-313X.2005.02376.x

17. Fu FQ, Mao WH, Shi K, Zhou YH, Asami T, Yu JQ. A role of brassinosteroids in early fruit development in cucumber. J Exp Bot. 2008;59:2299-2308. http://dx.doi.org/10.1093/jxb/ern093

18. Hanano S, Domagalska MA, Nagy F, Davis SJ. Multiple phytohormones influence distinct parameters of the plant circadian clock. Genes Cells. 2006;11:1381-1392. http://dx.doi. org/10.1111/j.1365-2443.2006.01026.x

19. Krishna P. Brassinosteroid-mediated stress responses. J Plant Growth Regul. 2003;22:289-297. http://dx.doi.org/10.1007/s00344-003-0058-Z
20. Janeczko A, Filek W, Biesaga-Kościelniak J, Marcińska I, Janeczko Z. The influence of animal sex hormones on the induction of flowering in Arabidopsis thaliana: comparison with the effect of 24-epibrassinolide. Plant Cell Tissue Organ Cult. 2003;72:147-151. http://dx.doi. org/10.1023/A:1022291718398

21. Hu Y, Bao F, Li J. Promotive effect of brassinosteroids on cell division involves a distinct CycD3-induction pathway in Arabidopsis. Plant J. 2000;24:693-701. http://dx.doi.org/10.1046/j.1365-313x.2000.00915.x

22. Janeczko A, Skoczkowski A. Mammalian sex hormones in plants. Folia Histochem Cytobiol. 2005;43:71-79.

23. Yang XH, Xu ZH, Xue HW. Arabidopsis membrane steroid binding protein 1 is involved in inhibition of cell elongation. Plant Cell. 2005;17:116-31. http://dx.doi.org/10.1105/tpc.104.028381

24. Janeczko A, Oklešt'ková J, Siwek A, Dziurka M, Pociecha E, Kocurek $\mathrm{M}$, et al. Endogenous progesterone and its cellular binding sites in wheat exposed to drought stress. J Steroid Biochem Mol Biol. 2013;138:384-394. http://dx.doi.org/10.1016/j.jsbmb.2013.07.014

25. Janeczko A, Filek W. Stimulation of generative development in partly vernalized winter wheat by animal sex hormones. Acta Physiol Plant. 2002;24:291-295. http://dx.doi.org/10.1007/s11738-002-0054-0

26. Erdal S, Dumlupinar R. Mammalian sex hormones stimulate antioxidant system and enhance growth of chickpea plants. Acta Physiol Plant. 2011;33:1011-1017. http://dx.doi.org/10.1007/s11738-010-0634-3

27. Kislev KV, Tyunin AP, Karetin YA. Influence of 5-azacytidine and salicylic acid on demethylase gene expression in cell cultures of Vitis amurensis Rupr. Acta Physiol Plant. 2013;35:1843-1851. http://dx.doi. org/10.1007/s11738-013-1222-0

28. Fieldes MA, Schaeffer SM, Krech MJ, Brown JCL. DNA hypomethylation in 5-azacitidine-induced early-flowering lines of flax. Theor Appl Genet. 2005;111:136-149. http://dx.doi.org/10.1007/ s00122-005-2005-9

29. Poirier F, Bourin P, Bladier D, Joubert-Caron R, Caron M. Effect of 5 -azacytidine and galectin-1 on growth and differentiation of the human b lymphoma cell line bl36. Cancer Cell Int. 2001;1:2. http:// dx.doi.org/10.1186/1475-2867-1-2

30. Christman JK. 5-Azacytidine and 5-aza-2-deoxycytidine as inhibitors of DNA methylation: mechanistic studies and their implication for cancer therapy. Oncogene. 2002;21:5483-5495. http://dx.doi. org/10.1038/sj.onc.1205699

31. Kaminskas E, Farrel AT, Wang YC, Sridhara R, Pazdur R. FDA drug approval summary: azacitidine (5-azacytidine, vidaza ${ }^{\mathrm{Tx}}$ ) for injectable suspension. Oncologist. 2005;10:176-182. http://dx.doi.org/10.1634/ theoncologist.10-3-176

32. Kondo H, Ozaki H, Itoh K, Kato A, Takeno K. Flowering induced by 5-azacytidine, a DNA demethylating reagent in a short-day plant, Perilla frutescens var. crispa. Physiol Plant. 2006;127:130-137. http:// dx.doi.org/10.1111/j.1399-3054.2005.00635.x

33. Choi CS, Sano H. Abiotic-stress induces demethylation and transcriptional activation of a gene encoding a glycerophosphodiesterase-like protein in tobacco plants. Mol Genet Genomics. 2007;277:589-600. http://dx.doi.org/10.1007/s00438-007-0209-1

34. Ruiz-Garcia L, Cervera MT, Martinez-Zapater JM. DNA methylation increases throughout Arabidopsis development. Planta. 2005;222:301306. http://dx.doi.org/10.1007/s00425-005-1524-6

35. Burn JE, Bagnall DJ, Metzger JD, Dennis ES, Peacock WJ. DNA methylation, vernalization, and the initiation of flowering. Proc Natl Acad Sci USA. 1990;90:287-291. http://dx.doi.org/10.1073/pnas.90.1.287

36. Köhler C, Wolff P, Spillane C. Epigenetic mechanisms underlying genomic imprinting in plants. Annu Rev Plant Biol. 2012;63:331-352. http://dx.doi.org/10.1146/annurev-arplant-042811-105514

37. Ohad N, Yadegari R, Margossian L, Hannon M, Michaeli D, Harada JJ, et al. Mutations in FIE, a WD Polycomb group gene, allow endosperm development without fertilization. Plant Cell. 1999;11:407-416. http:// dx.doi.org/10.1105/tpc.11.3.407

38. Ohad N, Margossian L, Hsu YC, Williams C, Repetti P, Fischer RL. A mutation that allows endosperm development without 
fertilization. Proc Natl Acad Sci USA. 1996;93:5319-5324. http:// dx.doi.org/10.1073/pnas.93.11.5319

39. Murashige T, Skoog F. A revised medium for rapid growth and bio assays with tobacco tissue cultures. Physiol Plant. 1962;15:473-497. http://dx.doi.org/10.1111/j.1399-3054.1962.tb08052.x

40. StatSoft Inc. STATISTICA (data analysis software system) version 9.1. Tulsa, OK; 2010.

41. Kuta E, Rojek J, Pawełek-Skoczylas A, Ślązak B, Bohdanowicz J. Autonomous endosperm induction in cultured unpollinated ovaries is strongly species dependent. Acta Biol Cracov Ser Bot. 2009;51(1 suppl):19.

42. Żabicki P, Rojek J, Kapusta M, Kuta E, Bohdanowicz J. Effect of estrone on somatic and female gametophyte cell division and differentiation in Arabidopsis thaliana cultured in vitro. Modern Phytomorphology. 2014;5:25-30.

43. Iino M, Nomura T, Tamaki Y, Yamada Y, Yoneyama K, Takeuchi $\mathrm{Y}$, et al. Progesterone: its occurrence in plants and involvement in plant growth. Phytochemistry. 2007;68:1664-1673. http://dx.doi. org/10.1016/j.phytochem.2007.04.002

44. Ylstra B, Touraev A, Brinkmann AO, Heberle-Bors E, Tunen AJV. Steroid hormones stimulate germination and tube growth of in vitro matured tobacco pollen. Plant Physiol. 1995;107:639-643.

45. Faure JE, Rotman N, Fortuné P, Dumas C. Fertilization in Arabidopsis thaliana wild type: developmental stages and time course. Plant J. 2002;30:481-488. http://dx.doi.org/10.1046/j.1365-313X.2002.01305.x

46. He K, Gou X, Yuan T, Lin H, Asami T, Yoshida S, et al. BAK1 and BKK1 regulate brassinosteroid-dependent growth and brassinosteroidindependent cell-death pathways. Curr Biol. 2007;17:1109-1115. http://dx.doi.org/10.1016/j.cub.2007.05.036

47. Veit B. Determination of cell fate in apical meristems. Curr Opin Plant Biol. 2004;7:57-64. http://dx.doi.org/10.1016/j.pbi.2003.11.009

48. Curtis M, Grossniklaus U. Molecular control of autonomous embryo and endosperm development. Sex Plant Reprod. 2008;21:79-88. http:// dx.doi.org/10.1007/s00497-007-0061-9 